\title{
Self-Sustaining Vortices in Protoplanetary Disks: Setting the Stage for Planetary System Formation
}

\section{Zsolt Regály ( $\nabla$ regaly@konkoly.hu )}

Konkoly Observatory, Research Centre for Astronomy and Earth Sciences

\section{Kundan Kadam}

Konkoly Observatory, Research Centre for Astronomy and Earth Sciences

Cornelis Dullemond

Heidelberg University

\section{Article}

Keywords: Self-Sustaining Vortices, planet formation, Planetary System Formation

Posted Date: November 30th, 2020

DOI: https://doi.org/10.21203/rs.3.rs-109121/v1

License: (1) This work is licensed under a Creative Commons Attribution 4.0 International License.

Read Full License 


\title{
Self-Sustaining Vortices in Protoplanetary Disks: Setting the Stage for Planetary System Formation
}

\author{
Zsolt Regály and Kundan Kadam \\ Konkoly Observatory, Research Centre for Astronomy and Earth Science, \\ Konkoly-Thege Miklós 15-17, 1121, Budapest, Hungary* \\ Cornelis P. Dullemond \\ Zentrum für Astronomie, Heidelberg University, Albert Ueberle Str. 2, 69120 Heidelberg, Germany门
}

(Dated: November 16, 2020)

\begin{abstract}
The core accretion scenario of planet formation assumes that planetesimals and planetary embryos are formed during the primordial, gaseous phases of the protoplanetary disk. However, how the dust particles overcome the traditional growth barriers is not well understood. Recently proposed viscous ring-instability explains the concentric rings observed in protoplanetary disks by assuming that the dust grains can reduce the gas conductivity, which can weaken the magneto-rotational instability. We present an analysis of this model with the help of GPU-based numerical hydrodynamic simulations of coupled gas and dust in the thin-disk limit. During the evolution of the disk the dusty rings become Rossby unstable and break up into a cascade of small-scale vortices. The vortices form secularly stable dusty structures, where the rapid dust growth can occur via the streaming instability. The phenomenon of self-sustaining vortices is consistent with observational constraints of exoplanets and sets a favorable environment for planetary system formation.
\end{abstract}

Over the past few decades, more than 3200 confirmed exoplanets have been detected [69] and it seems that most low mass stars harbor planetary systems. During the star formation process, protoplanetary disks (PPDs) are inevitably formed as a consequence of the conservation of angular momentum and these are considered to be the cradles of planets. The widely accepted core-accretion paradigm considers the formation of such planets as a bottom up process, wherein the dust grains in the PPD grow into kilometer sized planetesimals [53]. Collision of planetesimals leads to the formation of planetary embryos and subsequently terrestrial planets or giant planet cores [33. However, despite the immense progress in this field, how the small sized dust grains in the protostellar disk grow to become planetesimals is still an open question. Several obstacles stand in the way which prevent the successful dust growth beyond approximately 1 meter in size. Most notably, the drift barrier caused by aerodynamic drag on dust particles, results in their radial drift and eventual engulfment by the central star [65]. The fragmentation barrier on the other hand tends to break up growing dust particles due to the large relative velocities 64]. The theoretical framework may be consistent, so long as some "lucky few" seed particles manage to escape these barriers to become planetesimals [66].

The role of vortices in the process of planet formation has been a subject of debate for a long time. An anticyclonic vortex can be sustained in a Keplerian disk and it naturally forms a pressure maximum at its center. As the dust particles are attracted towards the pressure maxi-

\footnotetext{
* regaly@konkoly.hu

† dullemond@uni-heidelberg.de
}

mum, vortices function as dust traps and can play an important role in planetesimal formation [6, 32. Simulations suggest that large-scale vortices can form in a PPD (e.g., at the outer boundary of the dead zone) and the resulting structures look remarkably similar to the asymmetries observed in sub-millimeter emission 49. The vortex formation can also occur due to the turbulence generated from vertical shear instability in PPDs [39, 51]

Recently, Dullemond \& Penzlin [17] performed a linear perturbation analysis and demonstrated that PPDs can be unstable to viscous ring-instability (VRI). Smallsized dust grains tend to strongly reduce the conductivity of the gas by adsorbing electrons and ions [28, 44, 54]. This can inhibit the magneto-rotational instability (MRI) which is thought to be the primary source of turbulence in the disk [5. This can lead to a positive feedback loop as follows. An initial increase in the dust density reduces the MRI viscosity, which in turn can lead to accumulation of gas in its vicinity due to the mismatch in the mass transfer rate. As the dust particles drift towards the resulting pressure maxima, they can amplify the initial perturbations into spontaneous formation of concentric rings [17. Thus, VRI may be a potential mechanism behind the grand design ring structures often observed in the sub-millimeter emissions of PPDs [4, 18].

In this letter, we present the results of the first coupled gas and dust simulations in the thin disk limit, that investigate the progression of the VRI in $1 \mathrm{D}$ and $2 \mathrm{D}$ models. Using GPU-based hydrodynamic code GFARGO2, we show that dusty rings formed via VRI become Rossby unstable and break up into a multitude of clumps, which develop into small-scale vortices. In Fig. 1 we demonstrate the contrast between 1D (neglecting the azimuthal components of hydrodynamic equations) and 2D (model V2, 

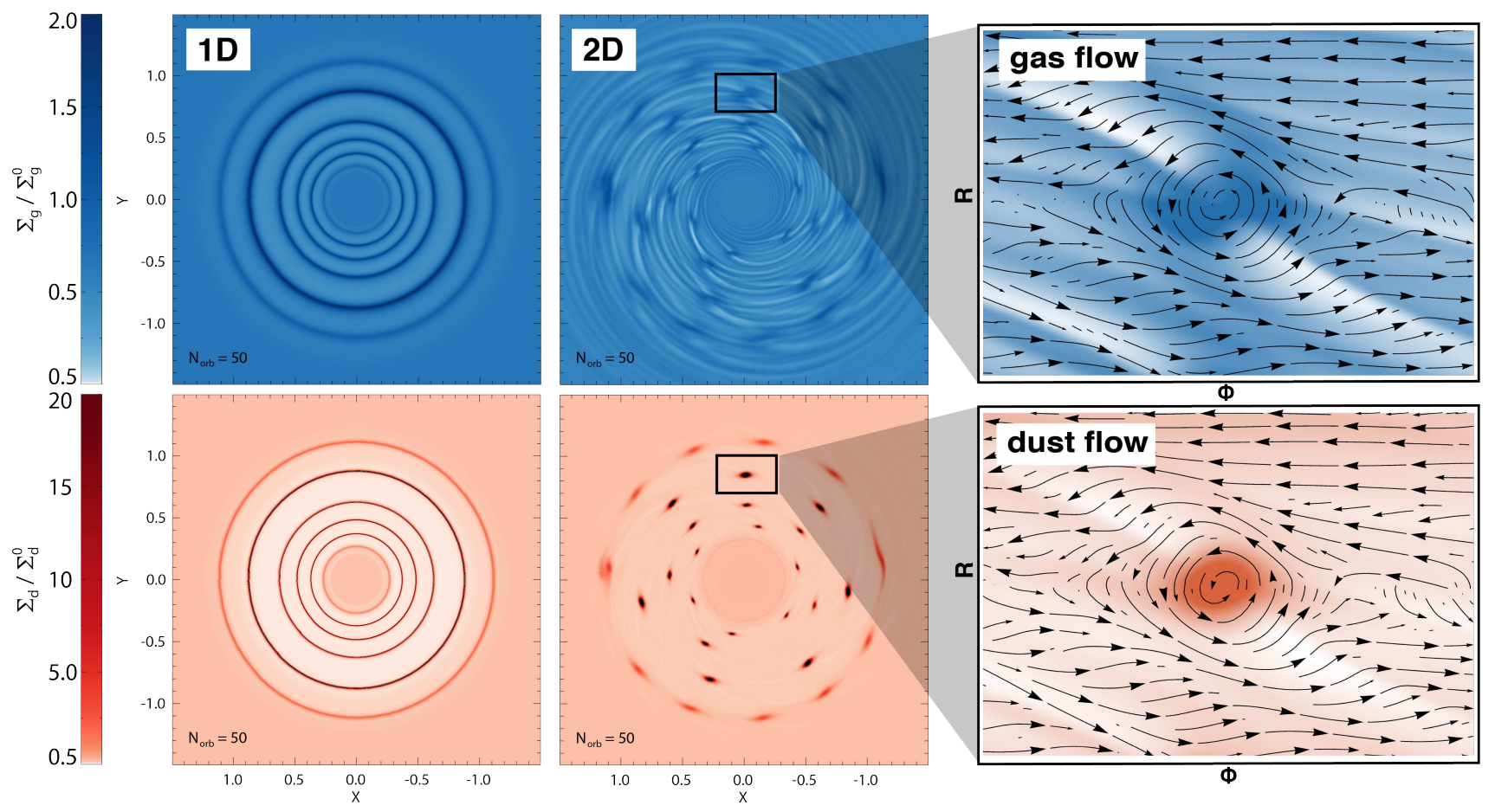

FIG. 1. Excitation of viscous ring instability in 1D (first column) and Rossby instability in 2D. Normalized gas and dust distributions at 50 orbits at 1 au are shown with blue and red colors. Rings developed in 1D model become Rossby unstable and brake up and form small-scale vortices in 2D simulations which allow for non-axisymmetry. The enlarged panels show gas and dust flow patterns in a frame of reference co-moving with the local Keplerian velocity, along with the respective surface density distributions.

explained later) simulations with the help of surface density distributions of dust and gas. Concentric rings form in the $1 \mathrm{D}$ model due to VRI, while in the $2 \mathrm{D}$ case, the rings become Rossby unstable. The flow patterns show that the regions of enhanced gas and dust are indeed anticyclonic vortices. The positive feedback loop responsible for VRI also makes the vortices self-sustaining and secularly stable. The vortices collect a significant amount of dust mass, while the midplane dust-to-gas density ratio grows beyond unity. These conditions are ideal for rapid dust growth and the formation of planetesimals via the streaming instability [42]. We emphasize that this is the first study to show that a large number of stable vortices can continually form in a PPD, as long as the dust is replenished from the outer regions. This phenomenon is consistent with several observational constraints such as enhanced planet formation in high metallicity PPDs [20] and high efficiency of the planet formation process [61. The self-sustaining vortices not only offer suitable conditions for planetesimal formation but also point towards the tantalizing idea of rapid, in situ formation of planetary cores.

In the outer disk at low densities, the ionization degree is much less sensitive to the dust properties [12]. This can be modelled with a larger value of the minimum viscosity in our simulations, in which case only VRI is excited without the secondary RWI. Thus, ring-like structures revealed by ALMA observations (e.g. HL Tau) are compatible with the parametric alpha model [18].

Here we mention some of the model details and initial conditions. The Shakura \& Sunyaev [55] $\alpha$ prescription for the disk viscosity was constructed such that it effectively depends on the dust-to-gas mass ratio $\left(\Sigma_{\mathrm{d}} / \Sigma_{\mathrm{g}}\right.$, see Methods for technical details). Gas and dust self-gravity, gas thermodynamics, and dust-feedback were taken into account in the numerical simulations. The dust was modeled as a pressureless fluid and its aerodynamic property (dust-gas coupling) was approximated by particles with a fixed Stokes number (St).

In this study, we primarily focus on the results of three simulations. We investigated the self-sustaining vortex formation in the terrestrial planet forming region of about 1 au (model V1) and in the outer disk at a distance of about 10 au (V2) as well as in high metallicity environment (V3). The initial distribution of the surface gas density followed a power law function of the disk radius, $\Sigma_{\mathrm{g}} \propto R^{-1 / 2}$. The initial dust-to-gas mass ratio was set uniformly to $1 \%$ and was increased 10 times for the high metallicity disk. In order to initiate VRI, an axisymmetric Gaussian perturbation was applied to the dust density at $1 \mathrm{au}$, with an amplitude of 10 percent above the background density and a half-width at half maximum of $0.001 \mathrm{au}$. 


\section{Viscous ring-instability and Rossby wave instability}

In all three simulation setups, we find that the protoplanetary disk exhibited VRI near the initial dust density perturbation, followed by development of RWI in the non-linear regime and vortex formation. We demonstrate the progression of this behavior qualitatively in Fig. 2 with the help of simulation V1. The figure shows the evolution of gas and dust density distributions in polar coordinates $(R-\phi$ plane).

Due to the positive feedback loop described earlier, the VRI was set in motion in the vicinity of the initial dust perturbation applied at $1 \mathrm{au}$. The VRI manifested as an axisymmentric ring in both dust and gas, increasing in magnitude over time, while also migrating inward due to the viscous evolution of the disk. In V1 simulation, VRI developed over the first 20 orbits $\left(N_{\text {orb }}\right)$. When the radial pressure gradient in this ring was sufficiently large, RWI was triggered. Panels a-1 and b-1 in Fig. 2 show the instance when the VRI became nonlinear and RWI clearly appeared in the simulation. A ring initially fragmented into dusty clumps with a typically large azimuthal mode number $(m \simeq 20)$. The dust accumulated at a faster rate than the gas, due to its strong affinity towards pressure maxima (see panel b-1). Panels a-2 and b-2 of Fig. 2 show the progression of RWI into several well-defined, anticyclonic vortices.

We emphasize that the vortices are self-sustained in such a way that the positive feedback loop between the dust accumulation, reduced viscosity, and the gas accumulation makes them secularly stable in a background of high viscosity. Note that in previous studies without this effect, the canonical values viscosity $\left(\alpha \approx 10^{-2}\right)$ as well as the backreaction of dust particles tended to smear out and destroy the vortices formed in a PPD [22]. The primary interaction between vortices was merger and it was reflected in a reduction in their number over time. The vortices were typically stable over thousands of orbits until they encountered the inner boundary. The gas distribution showed large scale spiral waves which originated at the location of each vortex. Rossby vortices have been shown to drive angular momentum transport in the disk 46. The resulting viscous torques can cause their orbital decay or inward migration, however, this phenomenon warrants further investigations.

The large scale spiral density waves associated with these vortices have a very interesting consequence. The waves can interfere constructively at a different radius, either inside or outside, giving rise to perturbations in gas surface density. With the positive feedback loop mentioned above, these gas enhancements can collect dust and result in the formation of a new "generation" of vortices. Thus, we term the original set of vortices formed at the location of the ring-instability as the first generation. Panels a-2 and b-2 of Fig. 2 depict the formation of a second and third generations of vortices, clustered at the radius of about 0.6 and $1.2 \mathrm{au}$. The last two columns of Fig. 2 show maturation of the second and third genera- tion vortices after mergers and inward migration, as well as formation of next generations of vortices. Note that it is also possible that VRI can form multiple rings first, which subsequently become Rossby unstable.

\section{Vortex evolution in terrestrial planet forming region}

In order to quantify the collective behavior of the vortices formed within the disk, we used a standard algorithm to find the location and area occupied by each vortex (see Methods). Consider the first column in Fig. 3 , depicting the temporal evolution of the disk with respect to the vortex properties for V1 model. The simulation focuses on approximately $1 \mathrm{au}$, which is the terrestrial planet forming region of a PPD. Panel a shows the total number of vortices identified on the disk $\left(N_{\text {vort }}\right)$ as a function of time, while panel $\mathrm{b}$ shows the spacetime diagram of the radial distance evolution of each vortex. The multiple generations of vortices can be clearly seen in panel b, each corresponding to an abrupt increase in $N_{\text {vort }}$. The reduction in $N_{\text {vort }}$ over time is caused by vortex coagulations or mergers. As the disk evolved, the dust particles drifted inward and left the computational domain through the inner boundary. In addition, the dust was also collected by the vortices, resulting in a decline of the background dust content of the disk. Thus, the production of vortices stopped at about 500 orbits. The average rate of migration of the vortices $\left(\sim 5 \times 10^{-3} \mathrm{au} \mathrm{yr}^{-1}\right)$ was about an order of magnitude faster than the calculated dust drift speed at $1 \mathrm{au}$.

Panel c of Fig 3 shows the total mass of dust as well as gas accumulated in all the vortices, while panel $\mathrm{d}$ shows the same quantities averaged for a single vortex. The colored area between solid and dashed lines encompasses the maximum and minimum estimates of mass, respectively, based on the bounds for MMSN. For V1 model, note that, despite of the migration of the vortices, $m_{\mathrm{g}}$ per vortex remained remarkably constant. The average dust mass in a vortex increased marginally over time in the vicinity of about $0.1 M_{\oplus}$. Note that the evolution described here reflects a combination of events for a number of vortices. As soon as a vortex is formed with RWI, it starts collecting dust, while dust mass is lost during its inward migration due via the process of diffusion. During the merger of two vortices, the dust mass is approximately conserved. However, the resulting vortex looses dust at an increased rate, thus maintaining a balance between dust drift towards the pressure maximum in the vortex and its diffusion because of the increased dust surface density. Panel e shows the midplane volumetric dust-togas density ratio $\left(\rho_{\mathrm{d}} / \rho_{\mathrm{g}}\right)$ assuming vertical equilibrium in gas and solid settling. The "max" and "center" values are the maximum and average values of this quantity at the vortex location, respectively. The "avg" values are averaged over the vortex area and are thus the lower bounds. Here the blue color encompasses the area between the estimated maximum and minimum values. As seen in 

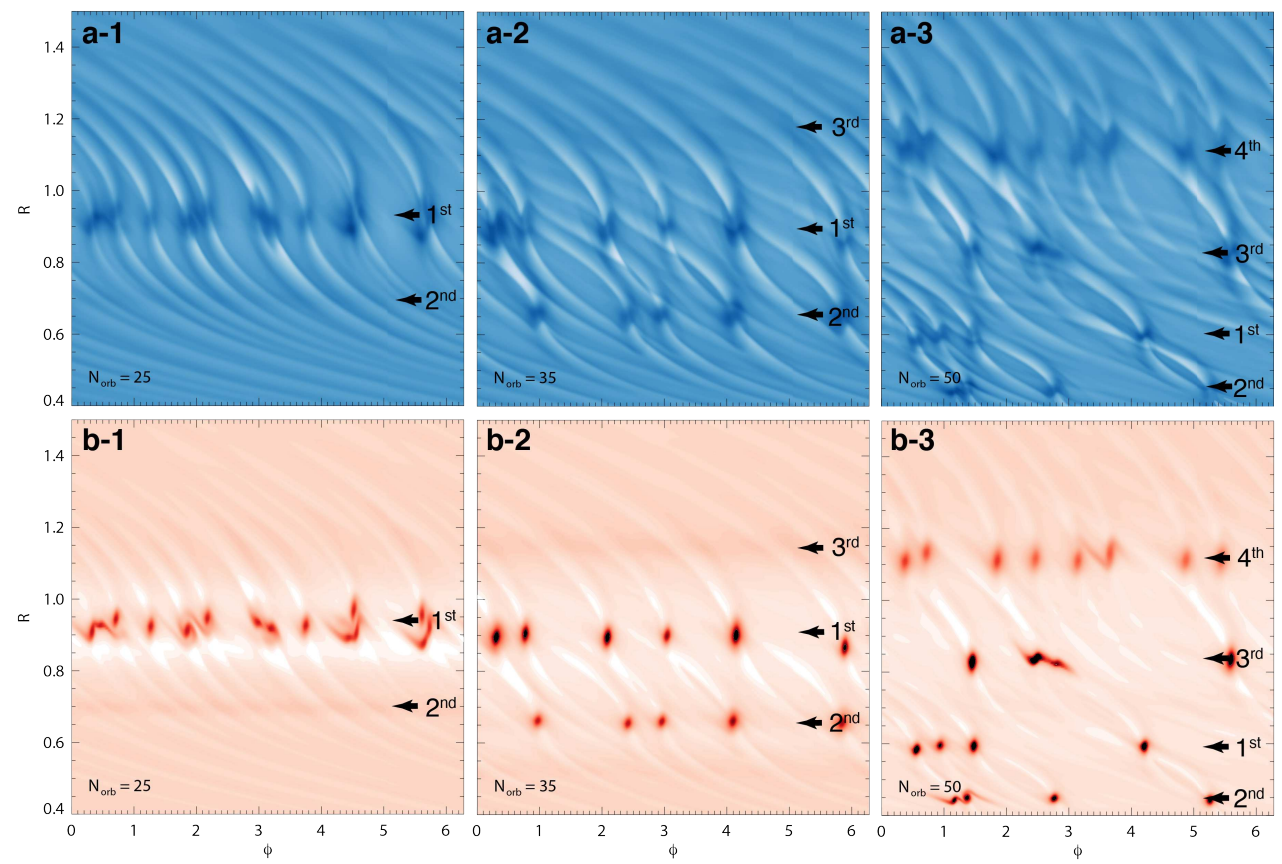
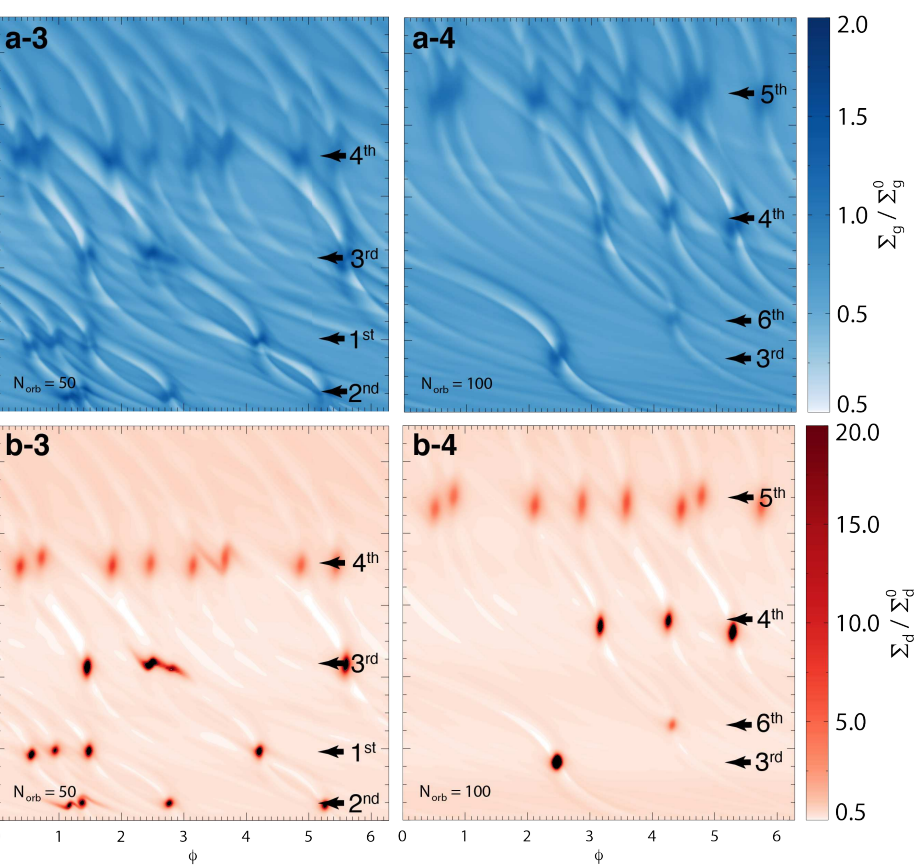

FIG. 2. Progression of self-sustaining vortex cascade in V1 model. The fields are sequential snapshots of the surface density distributions of gas (top) and dust (bottom), normalized with respect to the initial values and plotted in polar coordinates. The numbers on the right indicate the generation of the vortices, with the arrow pointing at their approximate radial position. The formation and orbital decay of successive generations as well as instances of vortex merging can be noticed.

panel e, the midplane dust density can be over 10 times larger than the gas inside a vortex. The corresponding dust-to-gas mass ratio often exceeded unity at the center of the vortices, indicating significant dust accumulation. Thus, although the vortices migrated at a faster rate, the local conditions could trigger streaming instability near the vortex centers and assist planetesimal formation [62]. The last panel $f$ shows the dust grain fragmentation radius $\left(a_{\text {frag }}\right)$ calculated with the typical assumptions for PPD [9] and using the higher mass bound for MMSN (Equation 22). The dust pebbles can achieve an approximate maximum size of $a_{\text {frag }}$ before they are destroyed through fragmentation resulting from high velocity collisions. With $a_{\text {frag }}$ often exceeding $1 \mathrm{~m}$, the vortices offer a more favorable environment for planetesimal formation, as compared to the traditional disk models [27.

\section{Vortex evolution in the outer disk}

A PPD can show significantly different behavior in the outer regions, where the disk self-gravity can affect its dynamical evolution. A sufficiently massive disk is prone to gravitational instability and fragmentation into gaseous clumps, due to the low values of Toomre's Q-parameter at large radii. In order to study the vortex cascade in the outer disk region, we conducted simulation V2 at a radial distance of approximately $10 \mathrm{au}$. In the case of model V2, the $\mathrm{Q}$ parameter dropped to $\sim 2$ in the vicinity of the vortices, implying that the self-gravity was significant in these regions. However, note that the Q-parameter remained above unity throughout this, as well as all simulations described in this study, indicating that the disks always remained gravitationally stable. We found that the disk self-gravity renders vortices more regularly spaced than in low-mass disk models (see Fig. 1).

Consider the second column in Fig. 3, which shows the temporal evolution of model V2. As seen in panel a, the number of vortices formed in each generation was about twice as many as compared to model V1. The inward migration rate of the vortices in the outer disk was about an order of magnitude slower $\left(\sim 2 \times 10^{-5} \mathrm{au} \mathrm{yr}^{-1}\right)$ as compared to the dust drift speed at the radius of $10 \mathrm{au}$. Thus, these vortices can offer sites for overcoming the dust drift barrier. The amount of gas as well as dust confined by vortices formed at the outer disk was similar to that found in terrestrial planet forming region (see panels b and c). As compared to the inner disk, the dustto-gas volumetric density ratio (panel e) was marginally larger in the outer than in the terrestrial planet forming region of $\mathrm{PPD}$. The maximum fragmentation size of the dust grains in the outer disk reached about half a meter. We hypothesize that both the increased number of vortices, their spatial distribution, as well as the enhanced concentration of the dust were assisted by the disk selfgravity. 
V1
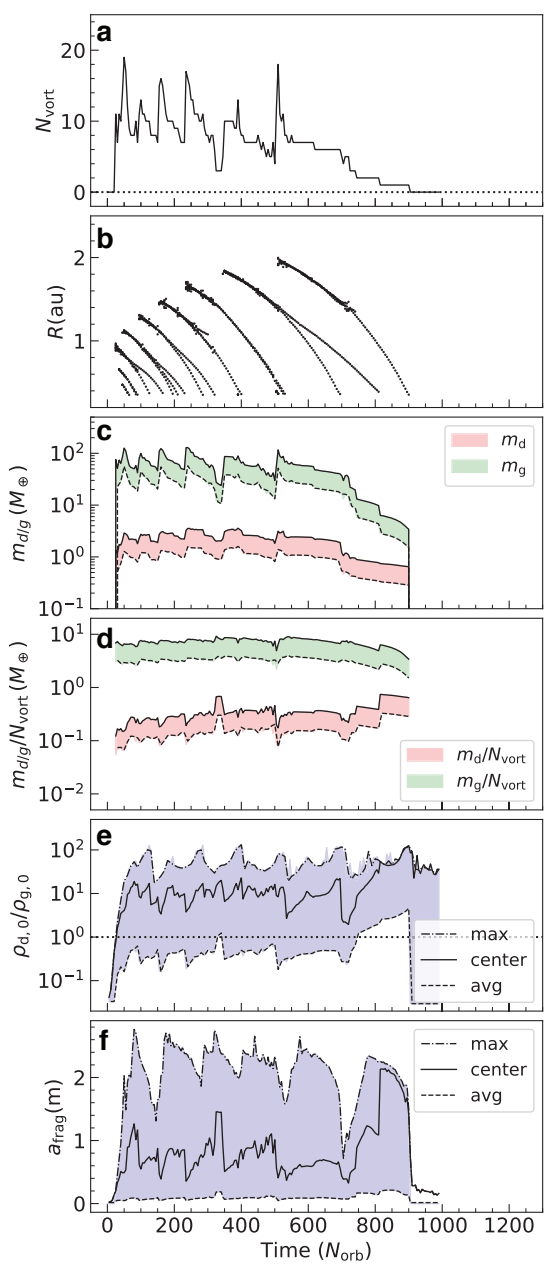

V2
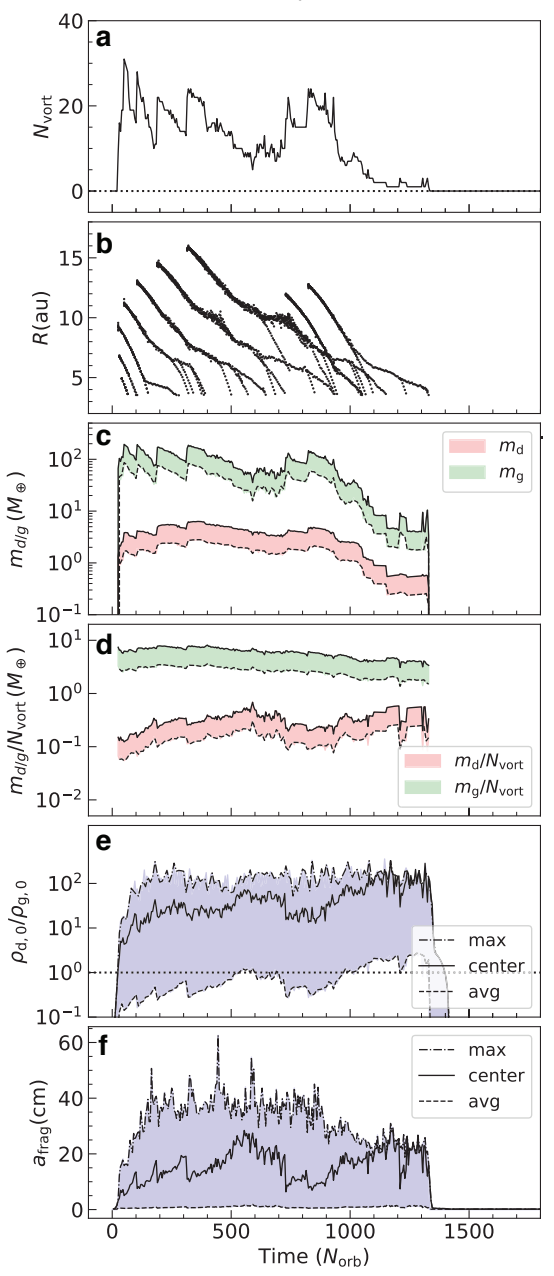

V3
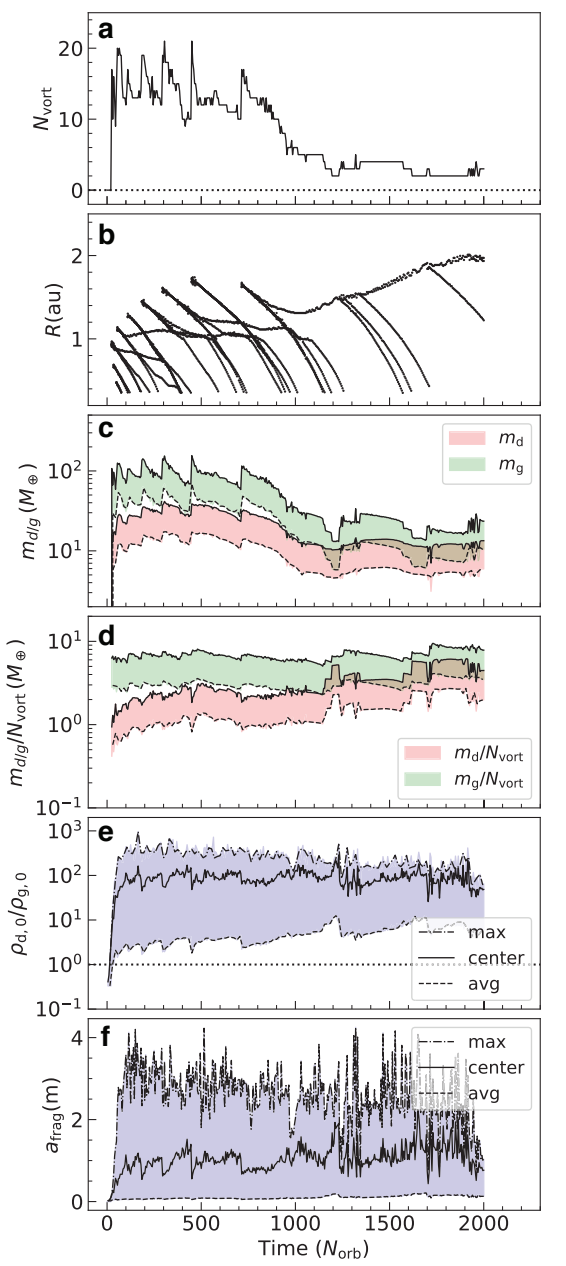

FIG. 3. Temporal evolution of the aggregate properties of the vortices formed in models V1 (terrestrial planet forming region), V2 (outer disk) and V3 (higher metallicity), conducted with $\mathbf{S t}=\mathbf{0 . 1}$. The rows show the following time-dependent physical parameters: (s) the number of vortices identified in the disk; (b) spacetime diagram of the radial distance evolution of the vortices; (c) the minimum and the maximum bounds of the total dust and gas mass inside vortices; (d) the average amount of dust and gas mass inside an individual vortex; (e) dust-to-gas volumetric density ratio at the disk midplane; (f) dust grain fragmentation radius. The shaded regions in panels $\mathrm{c}$ and $\mathrm{d}$ correspond to the maximum/minimum values calculated using the upper/lower bounds of gas density for the MMSN model. The dotted lines in panels e and f show the excitation criterion for triggering streaming instability and the meter size, respectively.

\section{Effect of stellar metallicity}

One of the major observations related to exoplanets statistics is that the frequency of giant planets is strongly correlated with the metallicity of the host star, presumably because of an enhanced rate of planetesimal formation in the disk [20, 24, 29]. We investigated the effect of stellar metallicity on the vortex formation with model V3, assuming 10 times greater initial dust mass distribution. Note that the limits of the effective $\alpha$ parameter were similar to the previous simulations, since this enhanced initial dust density $\left(\Sigma_{\mathrm{d}}^{0}\right)$ was used for its calculation (Equation 14). Consider the third column in Fig. 3 showing the temporal evolution of this high metallicity PPD. The dust back-reaction is known to shorten the vortex life-time [22], however, the stronger drag forces associated with higher dust content did not inhibit selfsustaining vortex formation (panel a) or shorten vortex lifetime (panel b). Both the total mass contained in the vortices as well as the average mass within a single vortex were about 10 times larger as compared to the solar metallicity counterpart (panels c and d). Similarly, the corresponding midplane dust-to-gas density ratio was an order of magnitude greater on average. Due to this larger density ratio, the viscosity in this region was also proportionally lower. The lower viscosity can explain the inefficiency of dust back-reaction in shortening the vortex lifetime. The fragmentation size of the dust particles was found to be about twice as large compared to the standard V1 model (panel e). Thus, the dynamics of the 
self-sustaining vortices was strongly affected by the stellar metallicity.

An interesting phenomenon was observed in model V3, wherein a mature vortex splits in two vortices during its evolution. The new vortex migrates inward while the progenitor migrates in the outward direction. This can be noticed in panel $\mathrm{b}$ of Fig. 3, with a bidirectional distance evolution beyond about 1000 orbits. A preliminary analysis indicates that such vortex splitting occurs due to the PPD's self-gravity, for reasons analogous to the phenomenon of vortex stretching [50].

\section{Vortex formation in the early and late stage PPDs}

As a PPD evolves, the dusty component of disk is subject to growth process, forming progressively larger sized particles. For investigating self-sustaining vortex formation in disks at different evolutionary stages, we conducted additional simulations (analogous to V1 model) assuming larger and smaller dust component with Stokes numbers of 1 and 0.001 , respectively. In order to excite RWI, a strong pressure gradient is required, which is reinforced by the dust enhancement in our model (see Equation (14). On the other hand, the turbulent diffusion of the dust component tends to inhibits its accumulation. The Stokes number of the dust particles directly affects both the diffusion coefficient as well as the dust backreaction (Equations 16 and 17, respectively), and thus has a significant impact on vortex formation.

For the model with $\mathrm{St}=1$, the turbulent diffusion of solids is practically negligible. This resulted in an enhanced vortex cascade and the number of vortices form was an order of magnitude larger $(\sim 150)$ than in V1 model, while the duration of the vortex phase was about twice as long. This indicates that the large-sized dust particles enhance the efficiency of the vortex formation process.

In the case of $\mathrm{St}=0.01$ and 0.001 models, where the dust particles are well coupled with the gas, selfsustaining vortices also developed. However, the vortex formation either required a lower background viscosity, e.g., $\alpha_{\mathrm{bg}}=10^{-3}$ or a stronger dust sensitivity of viscosity, (i.e., $\phi_{\mathrm{d}}=-2$, see Equation 14). The former can be explained by the fact that the strength of dust diffusion is proportional to the background viscosity (Equations 15 and 16). Extended regions of low effective viscosity can be found in the dead zone of a PPD where this condition may be satisfied. The small-sized dust grains can exhibit stronger recombination efficiency provided by the increased surface area per unit mass. Thus, a steeper dependence of viscosity on the dust content, i.e., a larger magnitude of $\phi_{\mathrm{d}}$, is indeed possible at small Stokes number.

Note that at low gas densities such as in the outer disk, the recombination is dominated by the gas phase and the ionization degree is much less sensitive to the dust properties [12. When this reduced sensitivity to the dust content is modeled with a larger value of the minimum viscosity, i.e. $\alpha_{\min } / \alpha_{\mathrm{bg}} \geq 0.9$, the vortex formation is suppressed and only VRI is excited. Thus, the observations of grand-scale, concentric rings in sub-millimeter wavelength emission [18] can be accounted for within this framework.

\section{Conclusion}

We studied a physically motivated model of PPD evolution wherein the MRI viscosity in the disk was suppressed with the local enhancement in the dust-to-gas ratio. The numerical experiments were carried out with the help of 2D global hydrodynamic simulations, which included the dust evolution as well as gas-dust coupling. The detailed results are presented for three scenarios- terrestrial planet forming region, the self-gravitating outer disk and a high metallicity PPD. We show that when the viscosity depends on the dust-to-gas mass ratio, the concentric rings formed due to viscous ring-instability [17] become Rossby unstable and as a result, the dusty ring breaks up into a multitude of small-scale, self-sustaining vortices. The vortices are secularly stable due to the positive feedback loop responsible for VRI and are subject to complex interactions such as mergers and orbital decay. An individual vortex could collect a significant amount of dust $\left(0.1-10 M_{\oplus}\right)$. A number of traditional barriers to the dust growth and planetesimal formation can be overcome inside the vortices due to the suitable local conditions as well as their stability over large number of orbits. Thus, the phenomenon of self-sustaining vortices sets a favorable stage for the formation of planetary systems with the core accretion scenario. Recent measurements show that protoplanetary disks around class II pre-main sequence stars do not contain enough dust mass needed to form the observed populations of exoplanet systems [61. This puts severe constraints on the timescale and efficiency on the planet formation process and the phenomenon of selfsustaining vortices may be a crucial piece of the puzzle.

As a final remark, in this study we used fixed Stokes numbers for the dust and therefore the processes of dust growth or fragmentation were neglected. Furthermore, the surface area available for ion and free electron recombination is smaller for grown dust as compared to small sized dust, and thus, the value of $\phi_{\mathrm{d}}$ in equation (14) can be size-dependent. Another theoretical challenge is that our study did not include an ab initio treatment of the ionization physics and should be followed up with comprehensive models containing relevant processes. Understanding the details of the vortex behavior such as their migration, merger and splitting properties also needs further investigations. 


\section{Methods}

\section{Hydrodynamical model}

We conducted the global 2D hydrodynamic simulations of the PPDs using GFARGO2, which is our GPU supported version of the FARGO code 40. In the gas-dust coupling, the dust was assumed to be a pressureless fluid. The dynamics of gas and dust components are described by the following equations:

$$
\begin{gathered}
\frac{\partial \Sigma_{\mathrm{g}}}{\partial t}+\nabla \cdot\left(\Sigma_{\mathrm{g}} \boldsymbol{v}\right)=0 \\
\frac{\partial \boldsymbol{v}}{\partial t}+(\boldsymbol{v} \cdot \nabla) \boldsymbol{v}=-\frac{1}{\Sigma_{\mathrm{g}}} \nabla P+\nabla \cdot \boldsymbol{T}-\nabla \Phi-\frac{1}{\Sigma_{\mathrm{g}}} \boldsymbol{f}_{\mathrm{drag}} \\
\frac{\partial e}{\partial t}+\nabla \cdot(e \boldsymbol{v})=-P \nabla \cdot \boldsymbol{v}+Q_{\nu}+Q_{ \pm} \\
\frac{\partial \Sigma_{\mathrm{d}}}{\partial t}+\nabla \cdot \Sigma_{\mathrm{d}} \boldsymbol{u}=-\nabla \cdot \boldsymbol{j} \\
\frac{\partial \boldsymbol{u}}{\partial t}+(\boldsymbol{u} \cdot \nabla) \boldsymbol{u}=-\nabla \Phi+\frac{1}{\Sigma_{\mathrm{d}}} \boldsymbol{f}_{\mathrm{drag}}-(\boldsymbol{u} \cdot \nabla) \boldsymbol{j}
\end{gathered}
$$

where $\Sigma_{\mathrm{g}}, \Sigma_{\mathrm{d}}$, and $\boldsymbol{v}, \boldsymbol{u}$ are the surface mass densities and velocities of gas and dust (being either dust particles or pebbles), respectively, and $e$ is the thermal energy density of the gas (per surface area). The gas pressure was given by

$$
P=(\gamma-1) e,
$$

where $\gamma=1.4$ is the adiabatic index of gas assumed to be $\mathrm{H}_{2}$ molecule.

The gravitational potential of the system in Equations (2) and (5) is

$$
\Phi_{\text {tot }}(R, \phi)=-G \frac{M_{*}}{R}+\Phi_{\text {ind }}(R, \phi)+\Phi_{\mathrm{sg}}(R, \phi),
$$

where the first term is the gravitational potential of the star in a given cell with radial distance $R$ and $G$ is the gravitational constant. Since the equations are solved in the cylindrical coordinate system centered on the star, Equation (7) includes the so-called indirect potential, $\Phi_{\text {ind }}(R, \phi)$ arising due to the displacement of the barycenter of the system caused by any disk non-axisymmetry (see its importance in, e.g., [50]). The indirect potential is calculated as

$$
\Phi_{\text {ind }}(R, \phi)=R \cdot G \int \frac{d m\left(\mathbf{R}^{\prime}\right)}{\mathbf{R}^{\prime \mathbf{3}}} \mathbf{R}^{\prime},
$$

which in the cylindrical coordinate system can be given as

$$
\begin{aligned}
\Phi_{\text {ind }}\left(R_{j}, \phi_{k}\right)= & R_{j} \cos \left(\phi_{k}\right) \sum_{j^{\prime}, k^{\prime}} G \frac{m_{j^{\prime}, k^{\prime}}}{R_{j}^{\prime 2}} \cos \left(\phi_{k^{\prime}}\right) \\
& +\sin \left(\phi_{k}\right) \sum_{j^{\prime}, k^{\prime}} G \frac{m_{j^{\prime}, k^{\prime}}}{R_{j}^{\prime 2}} \sin \left(\phi_{k^{\prime}}\right)
\end{aligned}
$$

where $m_{j, k}$ and $x_{j, k}, y_{j, k}$, are the mass and Cartesian coordinates of the grid cell $j, k$. To incorporate the effect of disk self-gravity, we calculate the gravitational potential of the disk, $\Phi_{\mathrm{sg}}$, by solving for the Poisson integral

$$
\begin{aligned}
\Phi_{\mathrm{sg}}(R, \phi)= & -G \int_{R_{\mathrm{in}}}^{R_{\mathrm{out}}} R^{\prime} d r^{\prime} \\
& \times \int_{0}^{2 \pi} \frac{\Sigma\left(R^{\prime}, \phi^{\prime}\right) d \phi^{\prime}}{\sqrt{R^{\prime 2}+R^{2}-2 R R^{\prime} \cos \left(\phi^{\prime}-\phi\right)}}(10)
\end{aligned}
$$

where $R_{\text {in }}$ and $R_{\text {out }}$ are the radial position of the disk inner and outer boundaries. This integral is calculated using a FFT technique which applies the two-dimensional Fourier convolution theorem for polar coordinates logarithmically spaced in the radial direction [see 8, Sect. $2.8]$.

In Equation (2) $\boldsymbol{T}$ is the viscous stress tensor of the gas,

$$
\boldsymbol{T}=\nu\left(\nabla \boldsymbol{v}+\nabla \boldsymbol{v}^{T}-\frac{2}{3} \nabla \cdot \boldsymbol{v} \boldsymbol{I}\right)
$$

whose components in polar coordinates are calculated according to 41. We used $\alpha$ prescription of Shakura \& Sunyaev [55] for the disk viscosity, $\nu$. In this case

$$
\nu=\alpha \frac{c_{\mathrm{s}}^{2}}{\Omega_{\mathrm{K}}},
$$

where $\Omega_{\mathrm{K}}=\left(G M_{*}\right)^{1 / 2} R^{-3 / 2}$ is the Keplerian angular velocity, $M_{*}$ is the mass of the central star, and the sound speed, $c_{\mathrm{s}}$, can be given as

$$
c_{\mathrm{s}}=\sqrt{\frac{\gamma(\gamma-1) e}{\Sigma_{\mathrm{g}}}} .
$$

The $\alpha$ parameter characterizing the strength of the magneto-rotational instability was parametrized using the prescription in [17] as

$$
\alpha=\alpha_{\mathrm{bg}}\left(\frac{\Sigma_{\mathrm{d}}}{\Sigma_{\mathrm{d}}^{0}}\right)^{\phi_{\mathrm{d}}}\left(\frac{\Sigma_{\mathrm{g}}}{\Sigma_{\mathrm{g}}^{0}}\right)^{\phi_{\mathrm{g}}},
$$

where $\Sigma_{\mathrm{g}}^{0}$ and $\Sigma_{\mathrm{d}}^{0}$ are the initial gas and dust densities. We limited the values of $\alpha$ between the background viscosity, $\alpha_{\mathrm{bg}}=10^{-2}$, and the MRI inactive viscosity, $\alpha_{\text {dead }}=10^{-4}$; the latter is approximately the minimum effective viscosity arising due to vertical shear instability [56]. In models $\mathrm{V} 1, \mathrm{~V} 2$, and $\mathrm{V} 3$ the values of $\phi_{\mathrm{d}}=-1$ and $\phi_{\mathrm{g}}=1$ were used, in which case $\alpha$ effectively varied in proportion to $\Sigma_{\mathrm{g}} / \Sigma_{\mathrm{d}}$ (i.e. $\partial \ln \alpha / \partial \ln \Sigma=-1$ ). For St $\leq 0.01$ cases, $\phi_{\mathrm{d}}=-2$ and $\phi_{\mathrm{g}}=1$ model was assumed.

The viscous heating $\left(Q_{\nu}\right)$ was taken into to account as described in [11. Other sources of heating and cooling, such as stellar and background irradiation, were modeled implicitly via $Q_{ \pm}$. We used $\beta$-cooling/heating prescription of 34 to let the gas release/gain its internal energy 
assuming $\beta=1$, i.e., the e-folding timescale of disk cooling/heating to the initial temperature is one orbit at all distances.

The turbulent diffusion of dust is modeled by the gradient diffusion approximation (see, e.g., [16, 43, 59]), wherein the diffusive flux, $\boldsymbol{j}$, is given as

$$
\boldsymbol{j}=-D\left(\Sigma_{\mathrm{g}}+\Sigma_{\mathrm{d}}\right) \nabla \frac{\Sigma_{\mathrm{d}}}{\Sigma_{\mathrm{g}}+\Sigma_{\mathrm{d}}} .
$$

Here, the diffusion coefficient of dust is defined as [63]

$$
D=\frac{\nu}{\left(1+\mathrm{St}^{2}\right)} \text {. }
$$

According to [7] the moment represented by the diffusive flux is taken into account in equation (5).

Equations (2) and (5) and are solved by a two-step method. First, the source term, i.e. the right-hand sides are calculated then it is followed by the conventional advection calculation. For the source term, we use a fully implicit scheme (see details in [57]). The drag force exerted by the gas on the dust is calculated as

$$
\boldsymbol{f}_{\mathrm{drag}}=\frac{\boldsymbol{v}-\boldsymbol{u}}{\tau_{\mathrm{s}}},
$$

where $\tau_{\mathrm{s}}=\mathrm{St} / \Omega$ is the stopping time. With this scheme, the effect of aerodynamic drag can be modeled for dust species that have stopping time that is much smaller than the time-step $\left(\tau_{\mathrm{s}} \ll \Delta t\right)$. For pebbles that have large stopping time $\left(\tau_{\mathrm{s}} \gg \Delta t\right)$, the method described is applicable as long as crossing orbits are not important for the dynamics. For simplicity, we assume that the dust has a fixed Stokes number.

The numerical domain extended between 0.5 and $2 \mathrm{au}$, with $N_{\mathrm{R}}=512$ logarithmically distributed radial and $N_{\phi}=1024$ equidistant azimuthal cells. Both the inner and outer edge the velocity components and density of gas are damped to the initial value according to the method described in [13].

The initial distribution of gas is set to a power-law function of $R$ as

$$
\Sigma_{\mathrm{g}}^{0}=\Sigma_{0} R^{-0.5}
$$

Since the disk self-gravity is neglected $\Sigma_{0}$ is a free parameter. Two possible disk masses were investigated by assuming $\Sigma_{0}=2000$ and $4500 \mathrm{~g} \mathrm{~cm}^{2}$, which covers the range for the canonical estimates for MMSN [1]. The initial dust-to-gas mass ratio was set uniformly to $\Sigma_{\mathrm{d}}^{0} / \Sigma_{\mathrm{g}}^{0}=10^{-2}$ in all models.

\section{Vortex analysis}

The following algorithm was used for the identification and analysis of the vortices formed in the disk. A vortex accumulates dust rapidly and thus creates an azimuthal asymmetry in the distribution of dust-to-gas mass ratio $\left(m_{\mathrm{d}} / m_{\mathrm{g}}\right)$. Thus, for detecting the location of all vortices at a given time, we identified local maxima in the field of $\left(m_{\mathrm{d}} / m_{\mathrm{g}}\right) /\left\langle m_{\mathrm{d}} / m_{\mathrm{g}}\right\rangle$. Here, in order to avoid abnormally large averages in the presence of a vortex, the denominator was azimuthally averaged at the given radius over the lowest half of the values. A region extending $10 \times 10$ grid cells in the $R-\phi$ plane was considered to have a local maximum if the difference between the maximum value and the minimum value within this region exceeds a certain threshold. The optimal value of the threshold was set by eye for each simulation -1.7 for $\mathrm{St}=0.1$ model and 2.2 otherwise. If two vortices were in close proximity, i.e., if the distance between them was less than $0.2 \times R$ and the radial separation $\Delta R<0.05$ in the units of au, they were deemed to be multiple detection of a single vortex. In such cases, the location of the maximum $\Sigma_{\mathrm{d}}$ was considered to be the center of the associated true vortex. The area occupied by a vortex was considered to be an ellipse in cylindrical coordinate system, centered at its location. The semi-major and semi-minor axes of such an ellipse were variable with radius, such that $a_{\theta}=0.24(R / 5)^{0.5}$ and $a_{R}=0.03(5 / R)^{0.8}$, where $R$ is in au. The area thus chosen was conservative, in order to include all of the dust accumulated inside a typical vortex. We rejected vortices with orbits less than 0.59 au because of the boundary effects. Note that the bulk properties of the vortices are not overly sensitive to the parameters chosen and we find this method to be sufficiently accurate.

The dust-to-gas volumetric density ratio is calculated as

$$
\frac{\rho_{\mathrm{d}}}{\rho_{\mathrm{g}}}=\frac{1}{\sqrt{2 \pi}} \Sigma_{\mathrm{d}} H_{\mathrm{d}} / \frac{1}{\sqrt{2 \pi}} \Sigma_{\mathrm{g}} H_{\mathrm{g}}
$$

where the gas pressure scale-height is calculated from the local sound speed given by Eq. (13) as

$$
H_{\mathrm{g}}=\frac{c_{\mathrm{s}}}{\Omega_{\mathrm{K}}} .
$$

To calculate the dust scale-height we assume size dependent vertical sedimentation for the dust, in which case

$$
H_{\mathrm{d}}=H_{\mathrm{g}} \sqrt{\frac{\alpha}{\mathrm{St}+\alpha}} .
$$

The dust grain fragmentation radius was calculated according to [9] as

$$
a_{\text {frag }}=\frac{2 \Sigma_{\mathrm{g}} v_{\text {frag }}^{2}}{3 \pi \rho_{\mathrm{s}} \alpha c_{\mathrm{s}}^{2}},
$$

with the typical assumptions of fragmentation velocity, $v_{\text {frag }}=10 \mathrm{~m} \mathrm{~s}^{-1}$, and the internal density of the dust aggregate, $\rho_{\mathrm{s}}=1.6 \mathrm{~g} \mathrm{~cm}^{-3}$, with $\Sigma_{\mathrm{g}}$ calculated using the higher bound for MMSN.

\section{ACKNOWLEDGMENTS}

The project was supported by the Hungarian OTKA Grant No. 119993. K.K. acknowledges funding from the 
European Research Council (ERC) under the European
Union's Horizon 2020 research and innovation program, grant No. 716155 (SACCRED).
[1] Adams, F. C. 2010, ARA\&A, 48, 47

[2] Ataiee, S., Pinilla, P., Zsom, A., Dullemond, C. P., Dominik, C., Ghanbari, J. 2013, A\&A, 553, 4

[3] Andrews S. M., et al., 2011, ApJ, 732, 42

[4] Andrews S. M., et al., 2018, ApJL, 869, L41

[5] Balbus S. A., Hawley J. F., 1998, RvMP, 70, 1

[6] Barge P., Sommeria J. 1995, A\&A, 295, L1

[7] Benítez-Llambay P., Krapp L., Pessah M. E., 2019, ApJS, 241, 25

[8] Binney, J. \& Tremaine, S. 1987, Princeton, N.J. : Princeton University Press, c1987.

[9] Birnstiel, T., Klahr, H., \& Ercolano, B. 2012, A\&A, 539, A148

[10] Crida, A., Morbidelli, A., Masset, F. 2006, 181, 587

[11] D'Angelo G., Kley W., Henning T., 2003, ApJ, 586, 540

[12] Dzyurkevich, N., Turner, N. J., Henning, T., et al. 2013, Astrophys. J., 765, 114

[13] de Val-Borro M., et al., 2006, MNRAS, 370, 529

[14] de Val-Borro, M., Artymowicz, P., D'Angelo, G., Peplinski, A. 2007, A\&A, 471, 1043

[15] Drążkowska, J., Alibert, Y., \& Moore, B. 2016, A\&A, 594, A105

[16] Dubrulle B., Morfill G., Sterzik M., 1995, Icar, 114, 237

[17] Dullemond C. P., Penzlin A. B. T., 2018, A\&A, 609, A50

[18] Dullemond C. P., et al., 2018, ApJL, 869, L46

[19] Dzyurkevich N., Turner N. J., Henning T., Kley W., 2013, ApJ, 765, 114

[20] Fischer, D. A., \& Valenti, J. 2005, Astrophys. J., 622, 1102

[21] Flock M., Turner N. J., Mulders G. D., Hasegawa Y., Nelson R. P., Bitsch B., 2019, A\&A, 630, A147

[22] Fu, W., Li, H., Lubow, S., Li, S. 2014, Astrophys. J., 788, 41

[23] Gammie C. F., 1996, ApJ, 457, 355

[24] Gonzalez, G. 1997, MNRAS, 285, 403

[25] Gonzalez J.-F., Laibe G., Maddison S. T., Pinte C., Ménard F., 2015, MNRAS, 454, L36

[26] Gonzalez J.-F., Laibe G., Maddison S. T., 2017, MNRAS, 467, 1984

[27] Heng, K. \& Kenyon, S. J. 2010, MNRAS, 408, 1476

[28] Ilgner M., Nelson R. P., 2006, A\&A, 445, 205

[29] Johansen, A., Youdin, A., \& Mac Low, M.-M. 2009, ApJL, 704, L75

[30] Kadam, K., Vorobyov, E., Regály, Z., et al. 2019, Astrophys. J., 882, 96

[31] Kanagawa K. D., Muto T., Tanaka H., Tanigawa T., Takeuchi T., Tsukagoshi T., Momose M., 2015, ApJL, 806, L15

[32] Klahr H. H., Henning T., 1997, Icar, 128, 213

[33] Kokubo, E., \& Ida, S. 1998, Icarus, 131, 171

[34] Les R., Lin M.-K., 2015, MNRAS, 450, 1503
[35] Li H., Finn J. M., Lovelace R. V. E., Colgate S. A., 2000, ApJ, 533, 1023

[36] Li H., Colgate S. A., Wendroff B., Liska R., 2001, ApJ, 551,874

[37] Li, H., et al. 2005, Astrophys. J., 624, 1003

[38] Lovelace R. V. E., Li H., Colgate S. A., Nelson A. F., 1999, ApJ, 513, 805

[39] Manger, N. \& Klahr, H. 2018, MNRAS, 480, 2125

[40] Masset F. S., 2000, ASPC, 219, 75

[41] Masset, F. S. 2002, A\&A, 387, 605

[42] Johansen, A., Klahr, H., \& Henning, T. 2011, A\&A, 529, A62

[43] Morfill G. E., Voelk H. J., 1984, ApJ, 287, 371

[44] Okuzumi S., 2009, ApJ, 698, 1122

[45] Okuzumi S., Momose M., Sirono S.-. iti ., Kobayashi H., Tanaka H., 2016, ApJ, 821, 82

[46] Paardekooper, S.-J., Lesur, G., \& Papaloizou, J. C. B. 2010, Astrophys. J., 725, 146

[47] Picogna G., Kley W., 2015, A\&A, 584, A110

[48] Pinilla P., Benisty M., Birnstiel T., 2012, A\&A, 545, A81

[49] Regály Z., Juhász A., Sándor Z., Dullemond C. P., 2012, MNRAS, 419, 1701

[50] Regály Z., Vorobyov E., 2017, A\&A, 601, A24

[51] Richard, S., Nelson, R. P., \& Umurhan, O. M. 2016, MNRAS, 456, 3571. doi:10.1093/mnras/stv2898

[52] Rossby, C.-G. et al., 1939, J. Marine Res., 2, 38

[53] Safronov, V. S. 1972, Evolution of the protoplanetary cloud and formation of the earth and planets

[54] Sano T., Miyama S. M., Umebayashi T., Nakano T., 2000, ApJ, 543, 486

[55] Shakura N. I., \& Sunyaev R. A. 1973, A\&A, 24, 337

[56] Stoll M. H. R., Kley W., 2016, A\&A, 594, A57

[57] Stoyanovskaya O. P., Vorobyov E. I., Snytnikov V. N., 2018, ARep, 62,

[58] Takahashi S. Z., Inutsuka S.-. ichiro ., 2014, ApJ, 794, 55

[59] Takeuchi T., Lin D. N. C., 2002, ApJ, 581, 1344

[60] Toomre, A. 1964, ApJ, 139, 1217

[61] Tychoniec, Ł., Manara, C. F., Rosotti, G. P., et al. 2020, arXiv e-prints, arXiv:2006.02812

[62] Youdin A. N., Goodman J., 2005, ApJ, 620, 459

[63] Youdin A. N., Lithwick Y., 2007, Icar, 192, 588

[64] Zsom, A., Ormel, C. W., Güttler, C., et al. 2010, A\&A, 513, A57

[65] Weidenschilling, S. J. 1977, MNRAS, 180, 57

[66] Windmark, F., Birnstiel, T., Güttler, C., et al. 2012, A\&A, 540, A73

[67] Wünsch R., Klahr H., Różyczka M., 2005, MNRAS, 362, 361

[68] Zhang K., Blake G., Bergin E., 2015, IAUGA, 29, 2256118

[69] http://exoplanets.org 


\section{Figures}
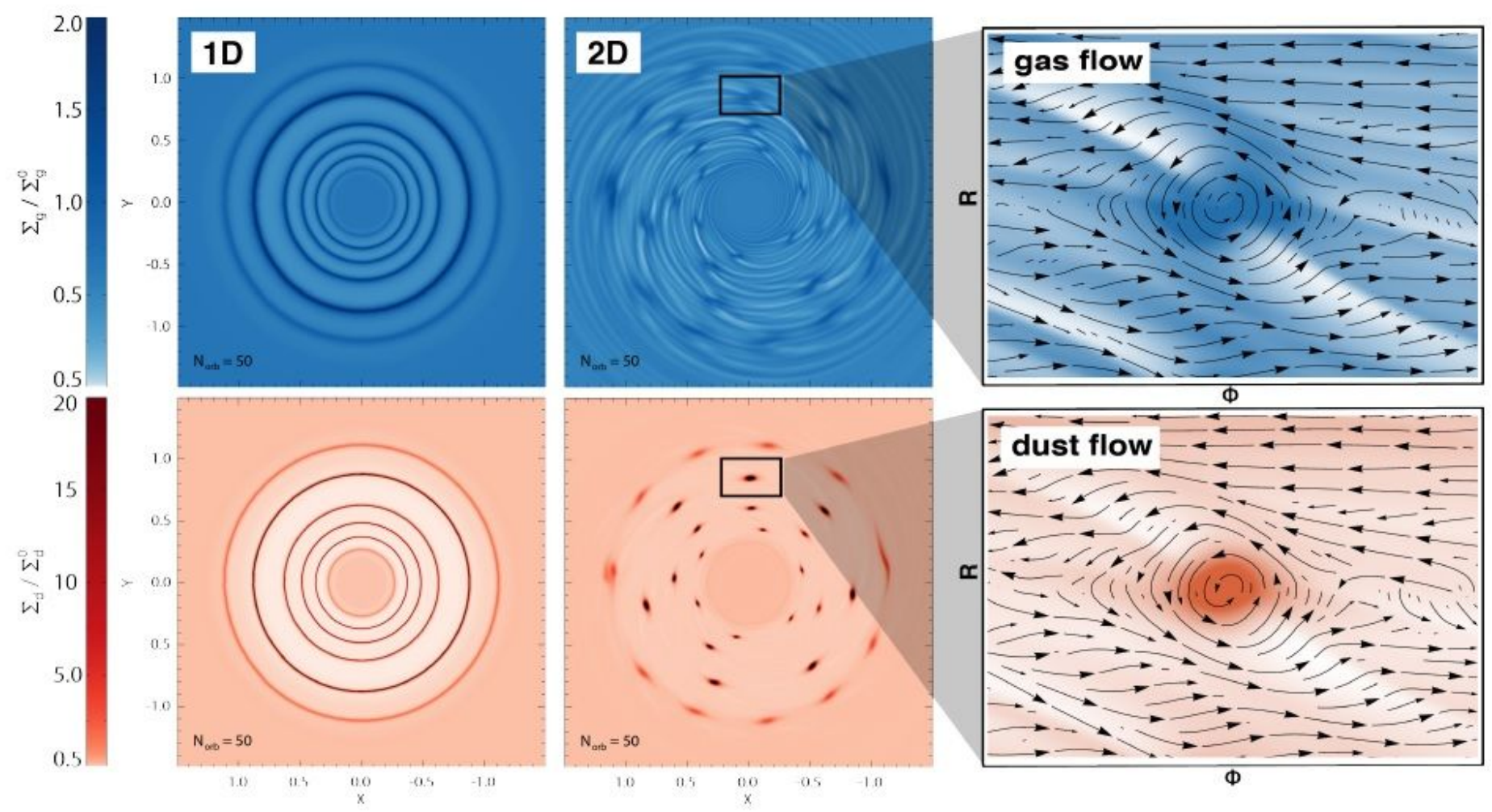

\section{Figure 1}

Excitation of viscous ring instability in 1D (first column) and Rossby instability in 2D. Normalized gas and dust distributions at 50 orbits at 1 au are shown with blue and red colors. Rings developed in 1D model become Rossby unstable and brake up and form small-scale vortices in 2D simulations which allow for non-axisymmetry. The enlarged panels show gas and dust flow patterns in a frame of reference comoving with the local Keplerian velocity, along with the respective surface density distributions. 

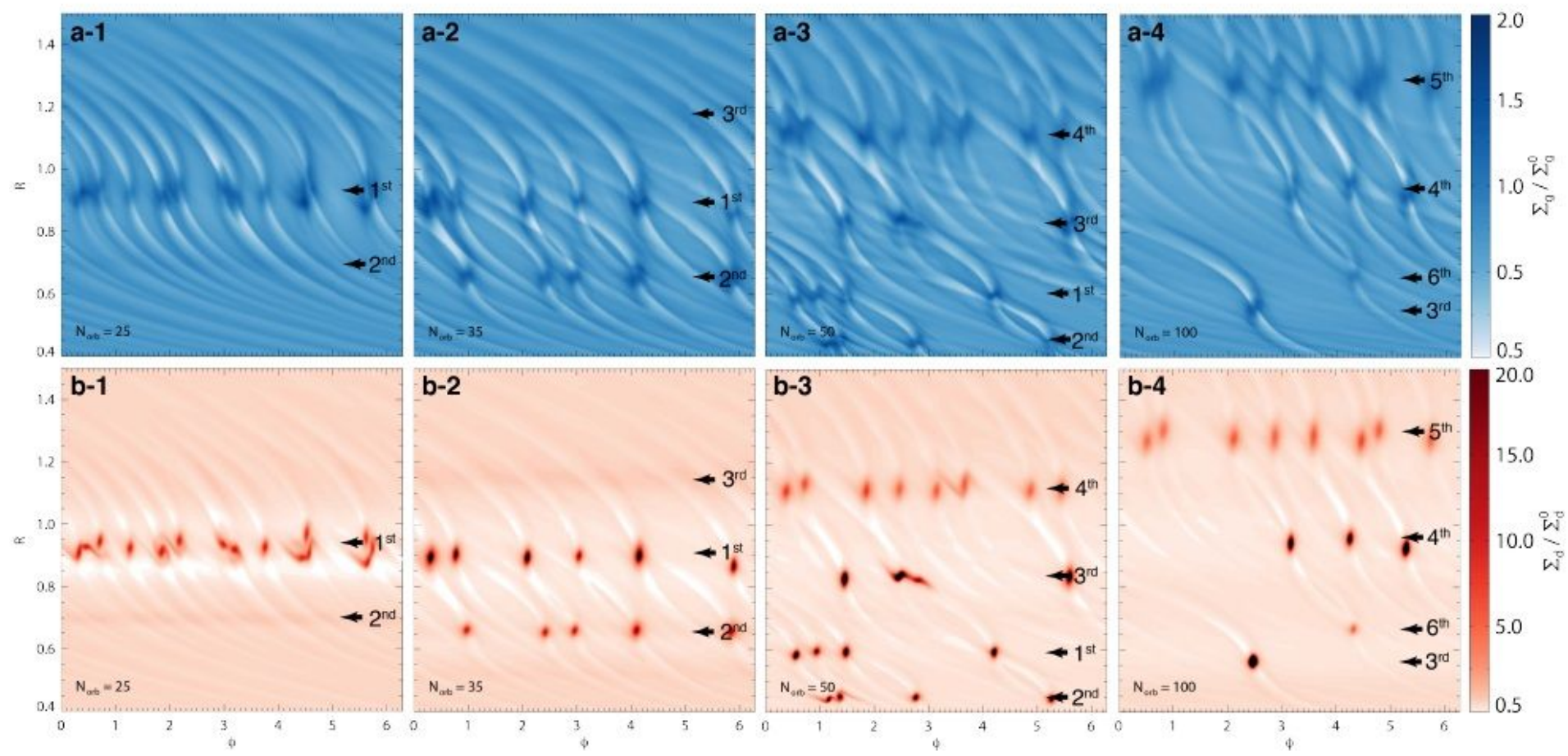

\section{Figure 2}

Progression of self-sustaining vortex cascade in V1 model. The fields are sequential snapshots of the surface density distributions of gas (top) and dust (bottom), normalized with respect to the initial values and plotted in polar coordinates. The numbers on the right indicate the generation of the vortices, with the arrow pointing at their approximate radial position. The formation and orbital decay of successive generations as well as instances of vortex merging can be noticed. 
V1
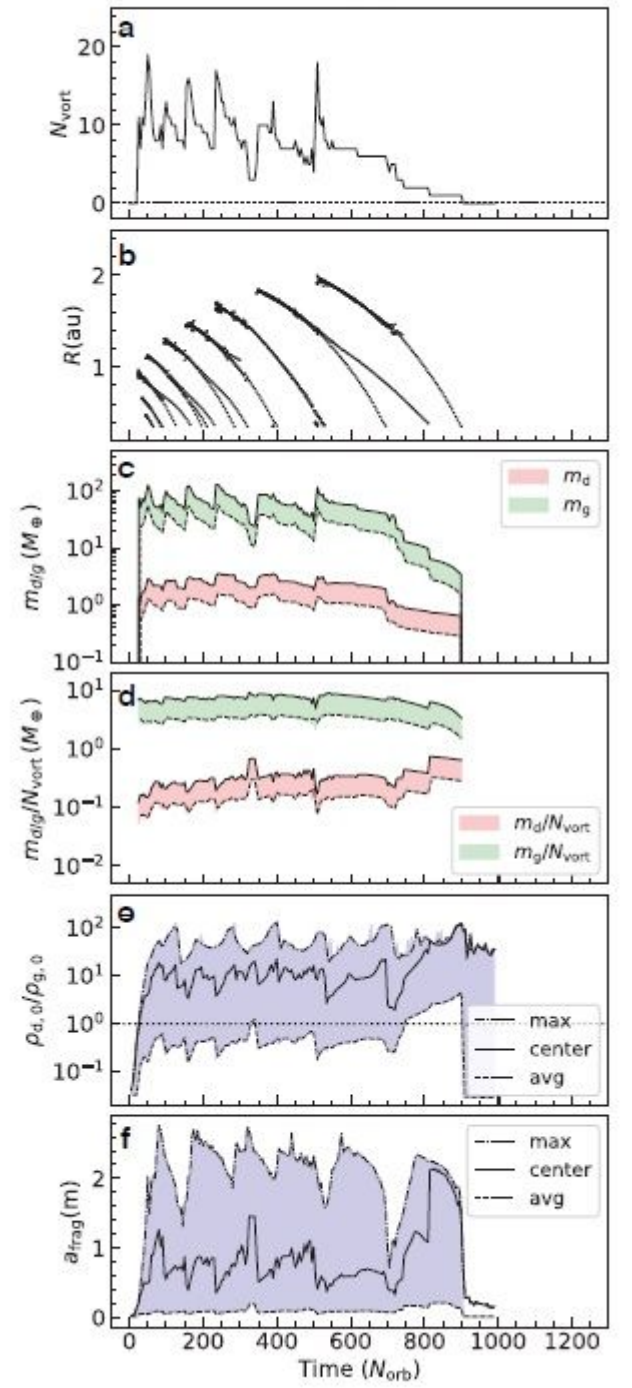

V2
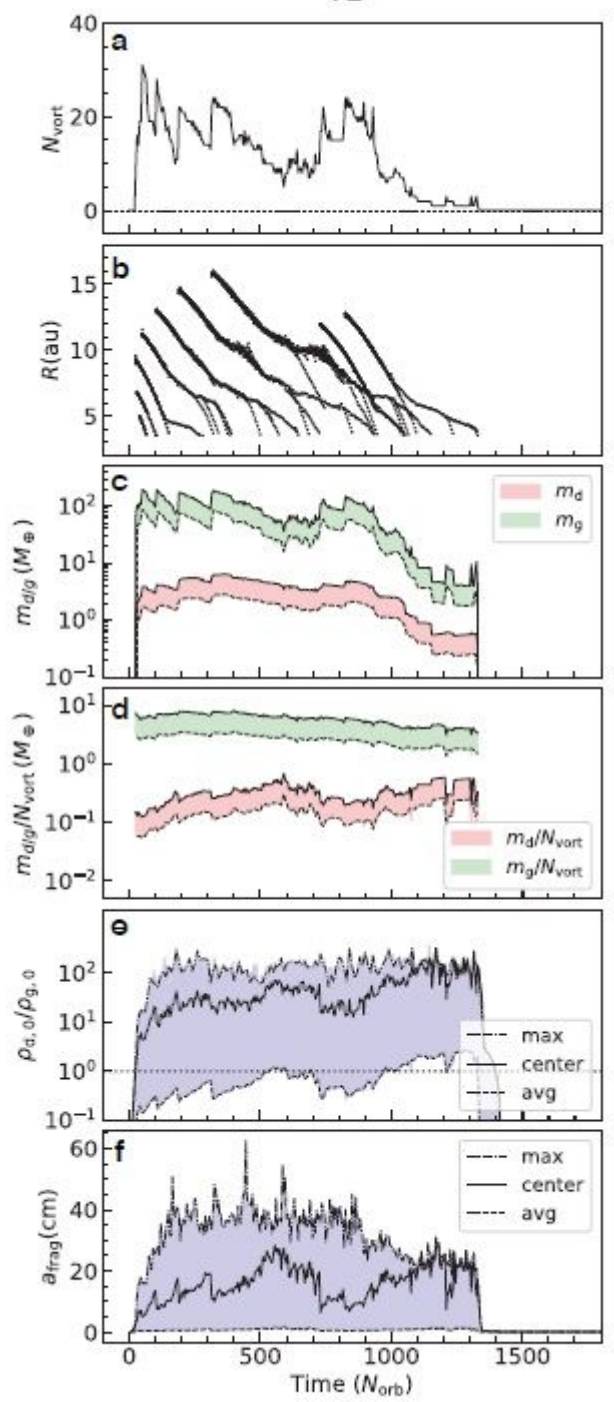

V3
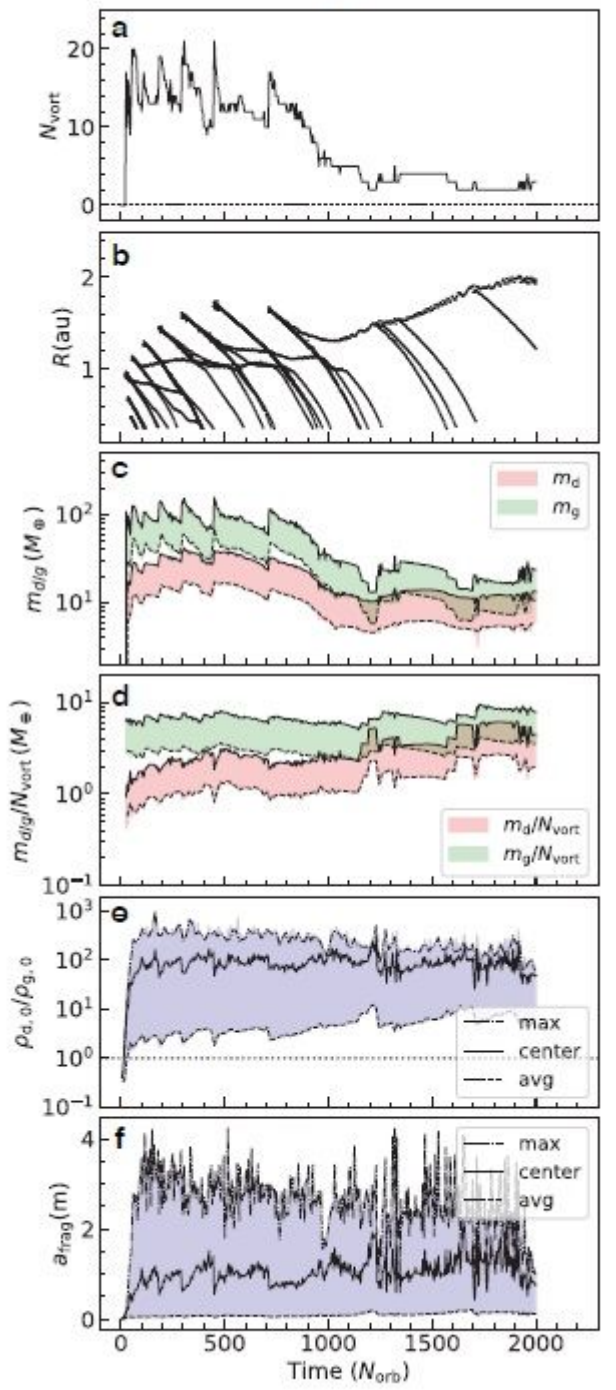

\section{Figure 3}

Temporal evolution of the aggregate properties of the vortices formed in models V1 (terrestrial planet forming region), V2 (outer disk) and V3 (higher metallicity), conducted with $\mathrm{St}=0.1$. The rows show the following time-dependent physical parameters: (s) the number of vortices identified in the disk; (b) spacetime diagram of the radial distance evolution of the vortices; (c) the minimum and the maximum bounds of the total dust and gas mass inside vortices; (d) the average amount of dust and gas mass inside an individual vortex; (e) dust-to-gas volumetric density ratio at the disk midplane; (f) dust grain fragmentation radius. The shaded regions in panels $\mathrm{c}$ and $\mathrm{d}$ correspond to the maximum/minimum values calculated using the upper/lower bounds of gas density for the MMSN model. The dotted lines in panels e and $f$ show the excitation criterion for triggering streaming instability and the meter size, respectively. 